\title{
RESTAURATOR
}

\section{INTERNATIONAL JOURNAL FOR} THE PRESERVATION OF LIBRARY AND ARCHIVAL MATERIAL

\section{EDITORS-IN-CHIEF}

Sigrid Eyb-Green, Vienna

Ute Henniges, Stuttgart

\section{EDITORIAL BOARD}

Mandana Barkeshli, Kuala Lumpur

Irene Brückle, Stuttgart

Salvador Muñoz Viñas, Valencia Andrea Pataki-Hundt, Cologne

Svetlana A. Dobrusina, St. Petersburg Paul Garside, London

Ulrike Hähner, Hildesheim José Luiz Pedersoli Júnior, Belo Horizonte Antje Potthast, Vienna

Theresa J. Smith, Buffalo

Christoph Krekel, Stuttgart

\section{DE GRUYTER SAUR}


ABSTRACTED/INDEXED IN AATA Online: Abstracts of International Conservation Literature $\cdot$ Baidu Scholar · Cabell's Directory · Chemical Abstracts Service (CAS): CAplus; SciFinder · CNKI Scholar (China National Knowledge Infrastructure) · CNPIEC · Compendex · Dimensions · EBSCO (relevant databases) $\cdot$ EBSCO Discovery Service $\cdot$ Engineering Village $\cdot$ Fluidex $\cdot$ Gale/Cengage $\cdot$ Genamics JournalSeek · Google Scholar · IBR (International Bibliography of Reviews of Scholarly Literature in the Humanities and Social Sciences) · IBZ (International Bibliography of Periodical Literature in the Humanities and Social Sciences) · Japan Science and Technology Agency (JST) · J-Gate · Journal Citation Reports/Social Sciences Edition · JournalGuide · JournalTOCs · KESLI-NDSL (Korean National Discovery for Science Leaders) · Microsoft Academic $\cdot$ Naviga (Softweco) · Primo Central (ExLibris) · ProQuest (relevant databases) $\cdot$ Publons $\cdot$ ReadCube $\cdot$ Reaxys $\cdot$ SCImago (SJR) $\cdot$ SCOPUS · Summon (Serials Solutions/ProQuest) · TDNet · Ulrich's Periodicals Directory/ulrichsweb · WanFang Data - Web of Science: Current Contents/Social and Behavioral Sciences; Social Sciences Citation Index $\cdot$ WorldCat (OCLC)

ISSN 0034-5806 · e-ISSN 1865-8431

All information regarding notes for contributors, subscriptions, Open access, back volumes and orders are available online at www.degruyter.com/restaur.

RESPONSIBLE EDITORS Dr. Sigrid Eyb-Green, Institut für Konservierung-Restaurierung, Akademie der bildenden Künste, Schillerplatz 3, A-1010 Wien, Email: s.eyb-green@akbild.ac.at

Dr. Ute Henniges, Staatliche Akademie der Bildenden Künste Stuttgart, Am Weißenhof 1, D-70191 Stuttgart, email: ute.henniges@abk-stuttgart.de

JOURNAL MANAGER Bendix Düker, De Gruyter, Genthiner Str. 13, 10785 Berlin, Germany, Phone: +49 (0)30 260 05-418, Fax: +49 (0)30 260 05-250, Email: bendix.dueker@degruyter.com

RESPONSIBLE FOR ADVERTISING Claudia Neumann, De Gruyter, Genthiner Straße 13, 10785 Berlin, Germany, Tel.: +49 (0)30 260 05-226, Fax: +49 (0)30 260 05-264,

Email: anzeigen@degruyter.com

(C) 2020 Walter de Gruyter GmbH, Berlin/Boston

B 43787

TYPESETTING Integra Software Services Pvt. Ltd., Pondicherry, India

PRINTING Franz X. Stückle Druck und Verlag e.K., Ettenheim

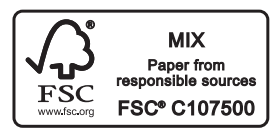





\section{Contents}

\section{Editorial}

Ute Henniges and Sigrid Eyb-Green

Editorial -1

\section{Original Works}

Lynn B. Brostoff and Cynthia Connelly Ryan

Tracing the Alteration of Verdigris Pigment through Combined Raman

Spectroscopy and X-ray Diffraction, Part I - 3

Joanna Karbowska-Berent, Izabela Żołowicz and Elżbieta M. Jabłońska

The Common Deathwatch Beetle Xestobium rufovillosum (DeGeer, 1774)

as a Pest for Paper in Books — 31

M. R. Singh and Deepakshi Sharma

Investigation of Pigments on an Indian Palm Leaf Manuscript (18th - 19th century)

by SEM-EDX and other Techniques — 49 